\title{
DEEP BRAIN TARGETING STRATEGY FOR BARE PARYLENE NEURAL PROBE ARRAYS
}

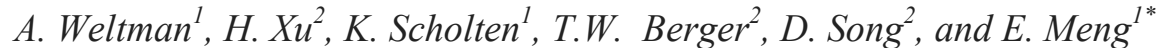 \\ ${ }^{1}$ University of Southern California, Viterbi School of Engineering, Los Angeles, California, USA \\ ${ }^{2}$ University of Southern California Center of Neural Engineering, Los Angeles, California, USA
}

\begin{abstract}
We present a Parylene neural microprobe array and the first demonstration of deep brain $(>5 \mathrm{~mm})$ implantation of bare polymer probes, enabled by a novel micropackaging technique. Targeting brain sites below the surface of the cortex with soft polymer probes typically requires bulky overcoats or insertion shuttles, which greatly increase probe dimensions and tissue damage, negating advantages of the flexible polymers. We achieved deep brain implantation of bare polymer probes by temporarily shortening their effective length using a biodegradable brace, thereby achieving the required transient increase in stiffness (Figure 1). This surgical approach solves a key technical obstacle preventing the widespread adoption of polymer microprobes. Here, we present mechanical, histological, and electrochemical evidence to support this approach.
\end{abstract}
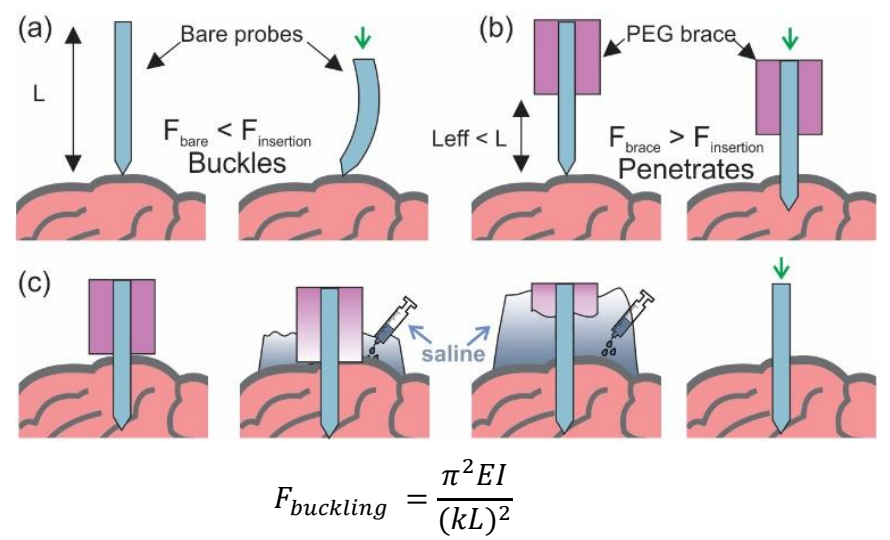

$$
F_{\text {buckling }}=\frac{\pi^{2} E I}{(k L)^{2}}
$$

Figure 1: (a) Buckling force threshold of bare probes is lower than that required for insertion, resulting in deformation. (b) PEG brace reduces the effective length of probes, increasing the buckling force threshold and enabling successful implantation. Buckling force $F$ is modeled by the equation above in which $k$ is the column effective length factor, $E$ is the Young's modulus, I is the moment of inertia, and $L$ is the probe's length. (c) Exposed probes are inserted to brain, PEG brace is dissolved in saline, and

\section{INTRODUCTION} probes inserted to desired depth $(5.5 \mathrm{~mm})$.

Soft polymers offer an appealing alternative to silicon, glass, or metal materials for the creation of neural probes. Rigid probes suffer inevitable signal degradation over time as chronic tissue irritation leads to an immune cascade that eventually may wall off of the implant $[1,2]$. Rigid probes have successfully achieved high-quality recordings on the scale of a few months, but this falls short of long-term goals desired to produce effective, life-long brain machine interfaces $[3,4]$. Whereas metals and silicon have Young's moduli on the order of hundreds of GPa, the stiffness of brain tissue is orders of magnitudes softer, at around $10^{-6} \mathrm{GPa}$. This disparity between the stiffness of brain tissue and implantable neural probes can lead to tissue damage as micro-motion of the brain causes chronic tissue irritation. It is speculated that the use of soft probes might mitigate this damage and attenuate the immune response and the glial cell sheath that impact achievable signal-to-noise ratios over time [5]. In one study, soft Parylene probes were shown to induce only a $12-17 \%$ neuronal loss around the implantation site compared to rigid silicon probes which incurred $40 \%$ neuronal loss at 4 weeks post-implantation [6].

The reduced stiffness of polymers, however, presents a technical challenge for surgical insertion into brain tissue. Polymer probes must be temporarily stiffened in order to penetrate brain tissue and for accurate surgical placement, typically via bulky biodegradable overcoats or insertion shuttles $[1,7,8]$ which can increase probe cross-section by $1.2-3,900 x$ 's thereby significantly adding to acute tissue injury $[1,9]$. The use of coatings or stiffeners can lead to other difficulties as well. Coatings may dull probe tips [9], cover electrode recording sites during surgery [7], or cause the probes to curl as the coating dries [10]. Retraction of a stiffener post-insertion may lead to the movement of a probe away from its target site, interfering with proper probe placement [8]. The need for temporary stiffening of probes during implantation has limited development of polymer probes to designs with short shanks (typically 1-2 mm), as shorter probes have larger buckling force thresholds, and has constrained recording sites to superficial cortical structures $[1,11,12]$. Our strategy overcomes these issues, and opens the door for the large scale acquisition of neural recordings from deep brain structures such as the cornu ammonis (CA1, CA3) and dentate gyrus (DG) in the hippocampus.

\section{METHODS}

Fabrication and design of Parylene neural probe array

Parylene microprobe arrays, consisting of a Parylene-metalParylene sandwich, were fabricated using standard micromachining techniques. A $10 \mu \mathrm{m}$ layer of chemical vapor deposited (CVD) Parylene forms the base of each probe. Platinum (Pt) electrode recording sites, traces, and contact pads are lithographically patterned on top of the base layer using sputter deposition at a thickness of 2,000 $\AA$, followed by lift-off. The metal layer is then encapsulated by a $10 \mu \mathrm{m}$ thick vapor deposited Parylene insulation layer. Electrodes and contact pads are exposed by deep reactive ion etching (DRIE) and the array outline is cut out from the substrate by means of DRIE as well. Devices are removed from the silicon carrier wafer by wetting them with water and gently lifting them with tweezers.

The microprobe array comprises eight probes with $250 \mu \mathrm{m}$ center-to-center spacing (5.5 mm long, $20 \mu \mathrm{m}$ thick, and 110-150 $\mu \mathrm{m}$ wide shanks; Figure 2), each with eight platinum (Pt) electrodes $(30 \mu \mathrm{m}$ exposed diameter). The electrodes are connected to contact pads by $5 \mu \mathrm{m}$ wide traces, with $5 \mu \mathrm{m}$ spacing. The array is designed to span a 2,000 $\mu \mathrm{m}$ anterior-posterior distance along a rat's hippocampus, with electrode groups positioned for simultaneous recording from two of the CA1, DG, or $\mathrm{CA} 3$ regions. Sham arrays lacking Pt electrode recording sites, traces, and contact pads were used for mechanical, in vitro, in vivo, and histological testing. 


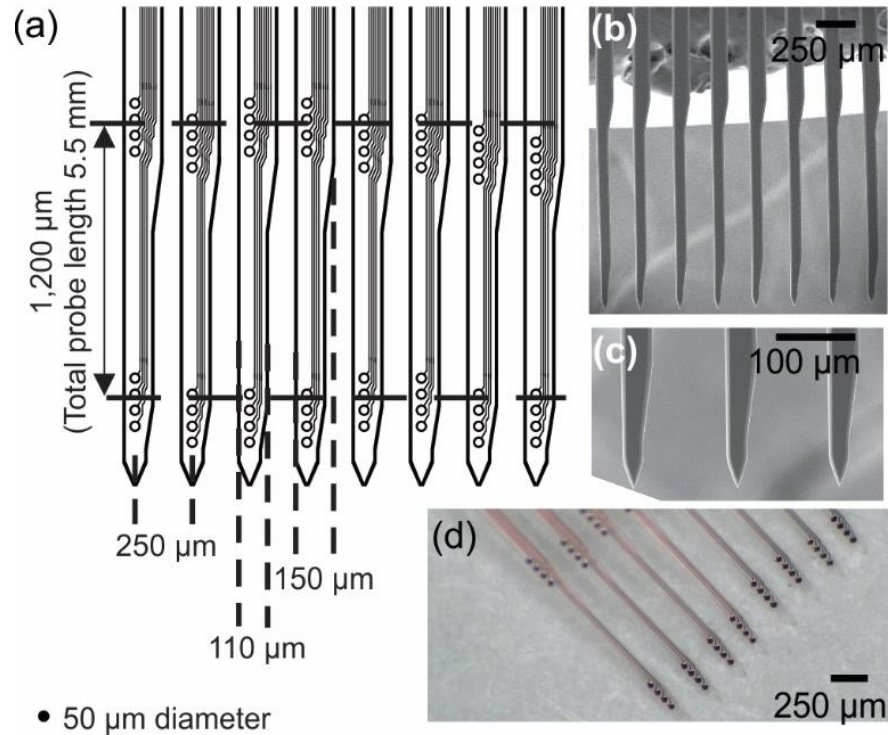

Figure 2: (a) Schematic showing dimensions of neural probe. Top group of four electrodes targets CA1 and the bottom group targets CA3 in the hippocampus (b) SEM of array (c) close-up of probe tips (d) micrograph close-up of fully fabricated array with Pt electrodes and traces.

\section{Micropackaging of probes for insertion}

Prior to surgical insertion, the array is partially encapsulated in a biodegradable polyethylene glycol (PEG) brace to halve the effective length of the probes and increase probe resistance to buckling (Figure 3). The PEG brace is created by sandwiching the probe array in between a two-part polydimethylsiloxane (PDMS) mold, which is then heated in a $50^{\circ} \mathrm{C}$ oven for 90 seconds. Molten PEG is injected into the cavity of the mold, and the mold is cooled for five minutes before the PEG brace encapsulated array is released from the PDMS mold.

The buckling force threshold of an individual probe can be mechanically modelled as a beam, and is directly proportional to the width $(w)$ and thickness $\left(t^{3}\right)$ of the probe, and inversely proportional to the probe length $\left(L^{2}\right)$, where $E$ is the Young's modulus of the probe material. (Eq. 1) Halving the effective length of the probe theoretically increases its buckling force threshold by a factor of four, which, when designed appropriately,
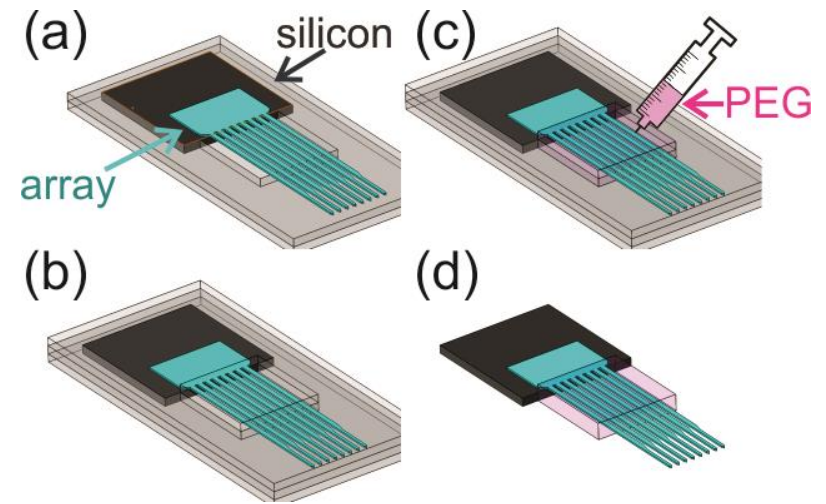

(d)

Figure 3: Fabrication of PEG brace: (a) probe array and silicon holder placed in bottom mold, (b) top mold placed over array, (c) heated PEG injected into mold, and (d) holder and braced array released. allows the buckling force threshold of the probe to surpass the force required to penetrate brain tissue. For our probes, modelled as beams that are clamped on one end and pinned on the other $(\mathrm{k}=$ 0.7 ) with a constant width of $150 \mu \mathrm{m}$ and a Young's modulus of $3.13 \mathrm{GPa}$ (data previously collected by lab), the calculated buckling force threshold of each probe is $0.21 \mathrm{mN}$ and $0.83 \mathrm{mN}$ with and without a PEG brace respectively. End supports of the probes were modelled as clamped by the PEG brace on one end and pinned by friction with the agarose or brain on the other end.

$$
F_{\text {buckling }}=\frac{\pi^{2} E w t^{3}}{5.88 L^{2}}
$$

\section{Experimental design}

In initial testing, the buckling force was directly measured for sham devices with and without the PEG encapsulation. Devices were driven into a metal plate using a computer controlled stepper motor until they buckled, while load was measured with a $50 \mathrm{~g}$ load cell (Omega, LCFA-50g). Buckling force was measured for arrays of 8 probes in addition to single probes. Values were compared to theoretical values calculated from beam theory and literature values $(\sim 1 \mathrm{mN})$ of required force for in vivo neural probe penetration [13].

In preparation for in vivo implantation, benchtop testing was conducted using brain phantoms $(0.5 \%$ agarose gel solution; Figure 4) to simulate the consistency and density of brain tissue. PEG encapsulated sham arrays were inserted into gel samples using a stereotaxic stage and the following technique (Figure 1): the exposed tips of the probes were slowly driven into the agarose up to an approximate depth of $2.75 \mathrm{~mm}$, at which point the PEG brace was dissolved with saline, and the remainder of the probe advanced to an ultimate depth of $5.5 \mathrm{~mm}$. Probe array were monitored during implantation for signs of buckling and deformation.

In subsequent in vivo rat studies, sham arrays encapsulated in the PEG brace were inserted into a male, Sprague-Dawley (SD) rat weighing between $300 \mathrm{~g}$ to $400 \mathrm{~g}$. Animal experiments were reviewed and approved by the Institutional Animal Care and Use Committee (IACUC) as well as the Department of Animal Resources of the University of Southern California (USC). During surgery, the superficial cortex above the dorsal hippocampi was exposed by removing a $2 \times 4 \mathrm{~mm}$ piece of cranium from each implantation site and carefully removing dura with forceps. Three screw holes were drilled into the cranium to serve as a port for anchor screws. The animal was anesthetized throughout the procedure, as confirmed by negative toe pinch withdrawal reflexes. The neural probe array was then inserted using the technique described above at a speed of $10 \mu \mathrm{m} / \mathrm{s}$, then the subject was perfused with paraformaldehyde and sacrificed immediately post-

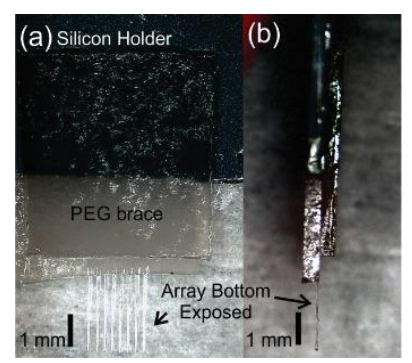

Figure 4: PEG brace for shortening effective length of probe, (a) top view and (b) side view.

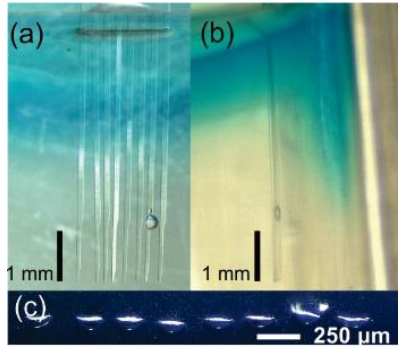

Figure 5: Braced array inserted into $0.5 \%$ agarose brain phantom :(a) front, (b) side, and (c) top view. 
surgery. Histology was performed following implantation and hippocampal slices (50 $\mu \mathrm{m}$ thick) were Nissl stained and examined by light-field microscopy to determine the acute damage incurred to neural tissue by implantation of the probe array.

Electrochemical testing of functional devices was performed via cyclic voltammetry to confirm electrode impedance values were sufficiently low for the resolvable acquisition of neural recordings.

\section{RESULTS}

The full array of eight probes, unbraced, buckled under a mean load of $1.26 \pm 0.19 \mathrm{mN}$, and were observed to consistently buckle during in vitro insertion tests with brain phantoms. The buckling threshold of a single probe could not be accurately recorded within the resolution of our load cell, but by extrapolating based on further experimentation, we estimated a single shank will buckle under a load of approximately $0.65 \mathrm{mN}$. As expected this is well below the loading anticipated during insertion [13], and consistent with the buckling witnessed during in vitro testing. Full arrays supported with a PEG brace had an average buckling force threshold of $4.24 \pm 0.24 \mathrm{mN}$, and for single braced-shanks buckling force threshold was measured directly as $2.14 \pm 0.25 \mathrm{mN}$. Figure 6 displays load measurements of repeated trials with a single shank supported by a brace and Figure 7 displays analogous data for full arrays with and without the brace.

Insertion of braced probes into $0.5 \%$ agarose brain phantoms during in vitro insertion tests confirmed what was expected following mechanical buckling measurements - that temporarily shortening shanks with the PEG encapsulation technique increased the buckling force threshold of probes and allowed for successful penetration of probes into agarose. During in vitro experiments arrays were inserted as described above and PEG braces dissolved within five minutes with repeat saline rinsing. Arrays were inserted into agarose without bending or buckling and could be placed accurately (Figure 5). The footprints made by the shanks in the agarose (stained blue in Figure 5, panel C) were no larger than the cross-sectional dimensions of our probes. This is a significant advantage compared to the enhanced cross-sectional insult to tissue caused by bulky overcoats and stiffeners.

Following benchtop demonstration, braced arrays were implanted into rat hippocampi. Braced arrays penetrated rat brain without buckling. One braced array experienced PEG softening upon exposure to ambient moisture causing the unsupported probes to buckle upon insertion, as visible in Figure 7. Thus, we directly observed that braced devices enable the successful penetration of rat brain, since unbraced devices buckled during insertion attempts.

Successful in vivo placement of the probe array in deep hippocampal layers was confirmed in Nissl stained histological slices (Figure 9). The stab wounds match probe cross-sectional dimensions and minimal damage to surrounding tissue was observed. This is a significant advantage over current insertion techniques which rely on bulky insertion aides add dramatically to the physical footprint of the neural probe.

Electrical impedance spectroscopy measurements from functional devices confirm low electrode impedance requisite for neural recordings $(<200 \mathrm{k} \Omega$ at $1 \mathrm{kHz})$ (Figure 8).

\section{DISCUSSSION}

Arrays encapsulated with a PEG brace were shown to successfully insert into a $0.5 \%$ agarose brain phantom as well as bilateral hippocampal sites in an anesthetized rat. This confirms the feasibility of our technique of temporarily shortening neural probes to achieve successful penetration of probes with longer shanks. Theoretically, this same strategy can be applied to probe

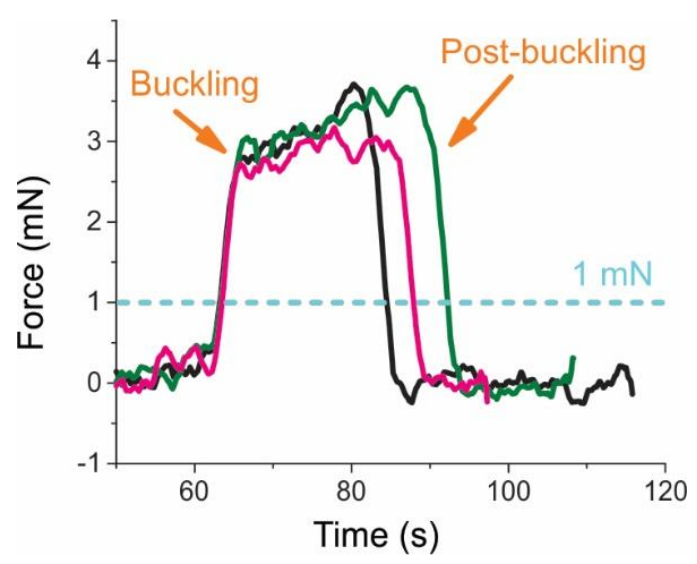

Figure 6: Load exerted on a single probe with PEG brace during induced buckling, three trials. Average buckling force threshold is $2.14 \pm 0.25 \mathrm{mN}$ (standard deviation) which exceeds the $1 \mathrm{mN}$ to penetrate brain tissue reported in literature.

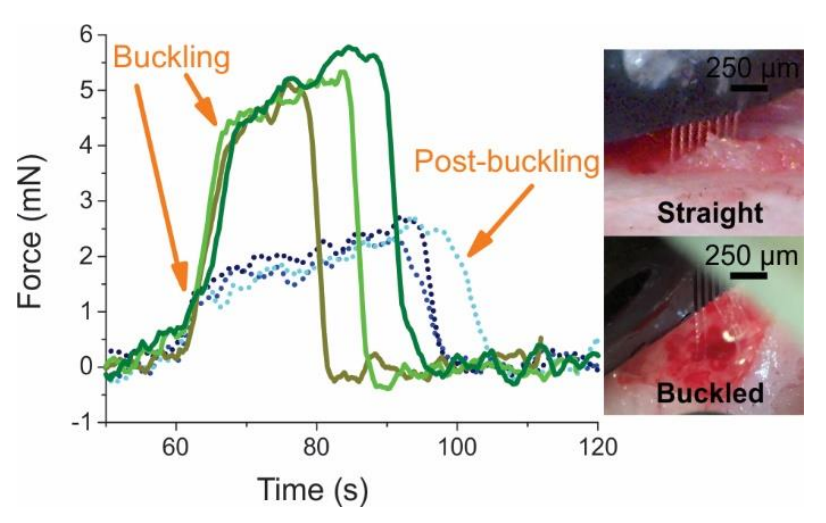

Figure 7: Load exerted on an 8 probe array with (solid curves) and without (dotted curves) PEG brace, three trials each. Arrays without PEG brace had an average buckling force threshold of $1.26 \pm 0.19 \mathrm{mN}$ and buckled during in vivo insertion, arrays supported with a PEG brace had an average buckling force threshold of $4.24 \pm 0.24 \mathrm{mN}$ and inserted without buckling.

shanks of any length, using an incremental PEG dissolution scheme if necessary to sequentially expose short probe segments.

Calculated and experimental buckling force measurements differed quite dramatically for probes with and without PEG brace encapsulation. A single, braced probe was estimated to have a buckling force threshold of $0.83 \mathrm{mN}$, but according to mechanical testing, had a higher threshold of $2.14 \mathrm{mN} \pm 0.25$. This discrepancy is curious, especially since the probe was approximated as a beam of constant width, which would have led to a higher estimate of the buckling force threshold. Choosing alternate end conditions for the buckling model (for example, modeling the beam as fixed on one end and free on the other end) would also only further decrease our estimation of buckling force threshold. Further study into this mismatch between expected and experimental buckling forces are warranted.

While PEG braces were created effectively using the two-part PDMS mold, heating of the PDMS in the oven sometimes resulted in contraction of the two thin PDMS layers, bending the probes in the mold. This difficulty was eliminated by lowering the PDMS heating time to 30 seconds instead of 90 seconds in order to prevent distortion of the PDMS layers. 


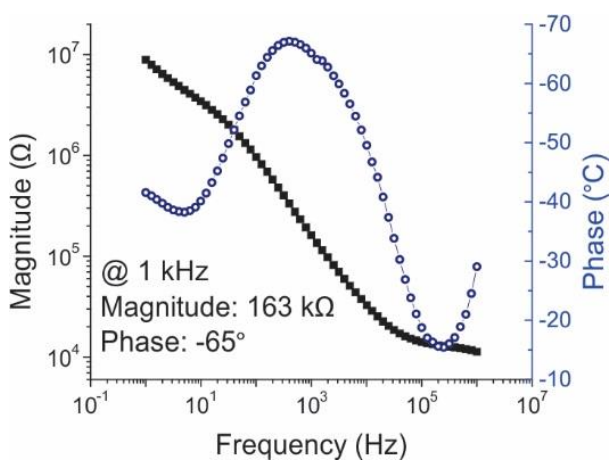

Figure 8: Electrode impedance spectroscopy (EIS) of Pt recording site. At $1 \mathrm{kHz}$, electrode has an impedance magnitude of $163 \mathrm{k} \Omega$ and a phase of $-65^{\circ}$.

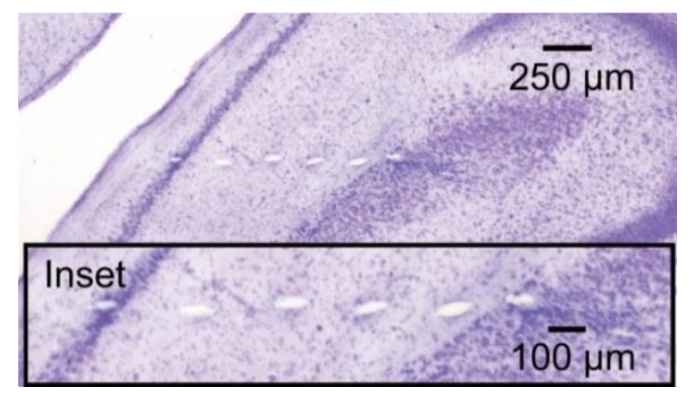

Figure 9: Nissl stained transverse slices of sham array insertion into left rat hippocampus and sacrificed immediately post-surgery. Slice depth $3.82 \mathrm{~mm}$.

Fully fabricated devices are being tested in insertion studies with the biodegradable PEG brace technique. Complete arrays with microfabricated metal electrodes, traces, and contact pads can experience curling do to internal material stresses upon release of the arrays from the silicon substrate. Such curvatures interfere dramatically with PEG encapsulation and insertion trials, as curved probes cannot be straightened during the PDMS mold, and enter agarose models at an angle, thereby missing their target destination and making precise positioning of electrodes in the hippocampal layer difficult. Different strategies to straighten the curved devices, including heat treatments and material etching to thin the probes in the direction of curvature, are being evaluated.

In on-going work, electrodes will be cleaned via cyclic voltammetry and checked with EIS to ensure intact traces prior to implantation. Arrays packaged with external connections will then be implanted in rats and the quality, chronicity, and density of electrophysiological recordings from active rat hippocampi will be evaluated and compared to their rigid, penetrating neural probe counterparts.

\section{ACKNOWLEDGEMENTS}

This work was funded by the NSF under award number CBET1343193. The authors would like to thank Dr. D. Zhu and the members of the USC Biomedical Microsystems Laboratory for their assistance. Travel support was provided by OAI. An OAI hybralign series 200 mask aligner was used for processing of Parylene devices.

\section{REFERENCES}

[1] S. Takeuchi, T. Suzuki, K. Mabuchi, and H. Fujita, "3D flexible multichannel neural probe array," Journal of Micromechanics and Microengineering, vol. 14, pp. 104-107, 2004.

[2] S. Takeuchi, D. Ziegler, Y. Yoshida, K. Mabuchi, and T. Suzuki, "Parylene flexible neural probes integrated with microfluidic channels," Lab Chip, vol. 5, pp. 519-23, May 2005.

[3] R. J. Vetter, J. C. Williams, J. F. Hetke, E. A. Nunamaker, and D. R. Kipke, "Chronic neural recording using silicon-substrate microelectrode arrays implanted in cerebral cortex," Biomedical Engineering, IEEE Transactions on, vol. 51, pp. 896-904, 2004.

[4] J. C. Williams, R. L. Rennaker, and D. R. Kipke, "Long-term neural recording characteristics of wire microelectrode arrays implanted in cerebral cortex," Brain Research Protocols, vol. 4, pp. 303-313, 1999.

[5] A. Mercanzini, K. Cheung, D. L. Buhl, M. Boers, A. Maillard, P. Colin, et al., "Demonstration of cortical recording using novel flexible polymer neural probes," Sensors and Actuators A: Physical, vol. 143, pp. 90-96, 2008.

[6] J. P. Seymour and D. R. Kipke, "Neural probe design for reduced tissue encapsulation in CNS," Biomaterials, vol. 28, pp. 3594-607, Sep 2007.

[7] D. Lewitus, K. L. Smith, W. Shain, and J. Kohn, "Ultrafast resorbing polymers for use as carriers for cortical neural probes," Acta biomaterialia, vol. 7, pp. 2483-2491, 2011.

[8] S. Felix, K. Shah, D. George, V. Tolosa, A. Tooker, H. Sheth, et al., "Removable silicon insertion stiffeners for neural probes using polyethylene glycol as a biodissolvable adhesive," in Engineering in Medicine and Biology Society (EMBC), 2012 Annual International Conference of the IEEE, 2012, pp. 871-874.

[9] P. Gilgunn, R. Khilwani, T. Kozai, D. Weber, X. Cui, G. Erdos, et al., "An ultra-compliant, scalable neural probe with molded biodissolvable delivery vehicle," in Micro Electro Mechanical Systems (MEMS), 2012 IEEE 25th International Conference on, 2012, pp. 56-59.

[10] T. D. Kozai, Z. Gugel, X. Li, P. J. Gilgunn, R. Khilwani, O. B. Ozdoganlar, et al., "Chronic tissue response to carboxymethyl cellulose based dissolvable insertion needle for ultra-small neural probes," Biomaterials, vol. 35, pp. 9255-68, Nov 2014.

[11] T. D. Kozai and D. R. Kipke, "Insertion shuttle with carboxyl terminated self-assembled monolayer coatings for implanting flexible polymer neural probes in the brain," J Neurosci Methods, vol. 184, pp. 199-205, Nov 152009.

[12] D. Ziegler, T. Suzuki, and S. Takeuchi, "Fabrication of flexible neural probes with built-in microfluidic channels by thermal bonding of Parylene," Journal of Microelectromechanical Systems, vol. 15, pp. 1477-1482, Dec 2006.

[13] B. Wester, R. Lee, and M. LaPlaca, "Development and characterization of in vivo flexible electrodes compatible with large tissue displacements," Journal of neural engineering, vol. 6, p. $024002,2009$.

\section{CONTACT}

*E. Meng, tel: +1-123-740-6952; ellis.meng@usc.edu 